\title{
Análisis fisicoquímico y microbiológico del proceso de co-compostaje a partir de biomasa de leguminosa y ruminaza
}

\section{Physicochemical and microbiological analysis of co-composting process from biomass legume and bovine rumen}
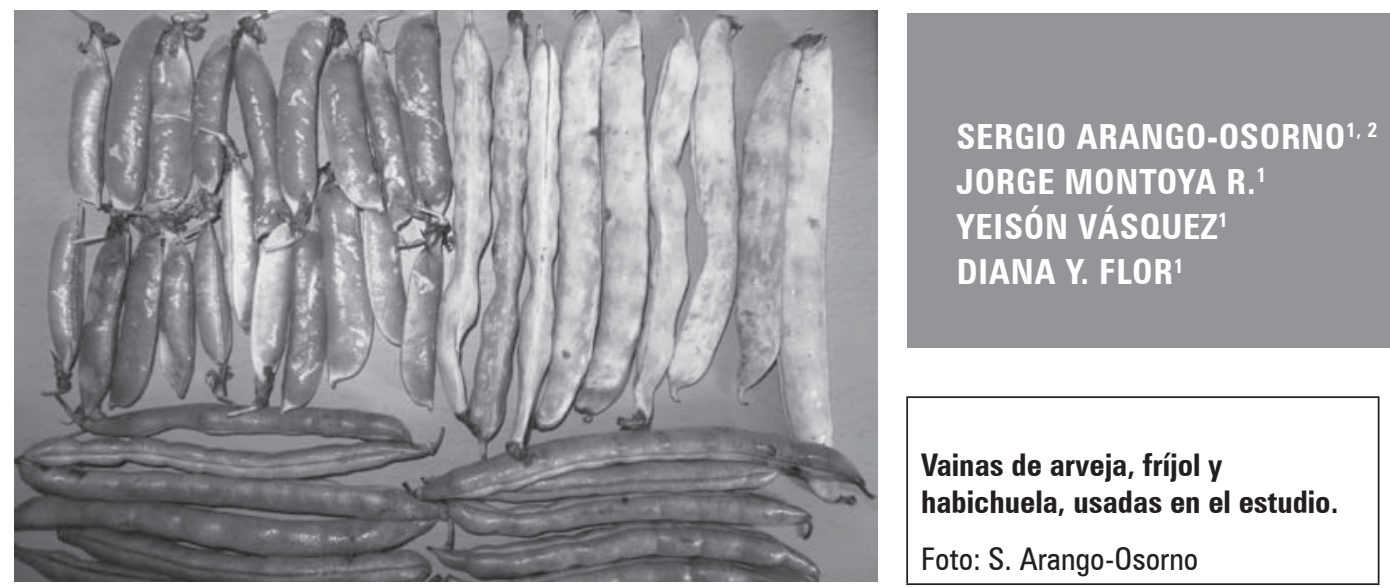

\section{RESUMEN}

En este artículo se presentan resultados de los análisis fisicoquímicos y microbiológicos individuales y combinados de compost obtenidos por codigestión aerobia de residuos de leguminosa y rumen bovino. La variable independiente fue el porcentaje en masa de residuos de leguminosa, obteniéndose cuatro tratamientos: 0, 25, 50 y $100 \%$. La fuente de leguminosa se obtuvo de residuos de vainas de fríjol al 58\%, arveja al $39 \%$ y habichuela al $3 \%$ en masa húmeda. La ruminaza fue obtenida del lavado estomacal de bovinos poco tiempo después del sacrificio del ganado en una planta industrial frigorífica. Se analizó la temperatura, pH, humedad, cenizas, densidad, conductividad, capacidad de retención de agua, capacidad de intercambio catiónico y elementos como C orgánico, N, K, Ca, Mg y Zn. La relación másica de carbono/nitrógeno (C/N) más baja de co-compostaje se encuentra en la mezcla leguminosa-ruminaza al $50-50 \%$ con 28,51, mientras que el compost individual vegetal de solo leguminosa fue de 9,14. Los parámetros de porcentaje en masa de N, Mg y Zn, así como la CIC (capacidad de intercambio catiónico) y conductividad presentaron los valores más altos en el compost vegetal, sin presencia de Salmonella, convirtiéndolo en una potencial enmienda orgánica que puede aportar dichos nutrientes al suelo en el cultivo que lo requiera.

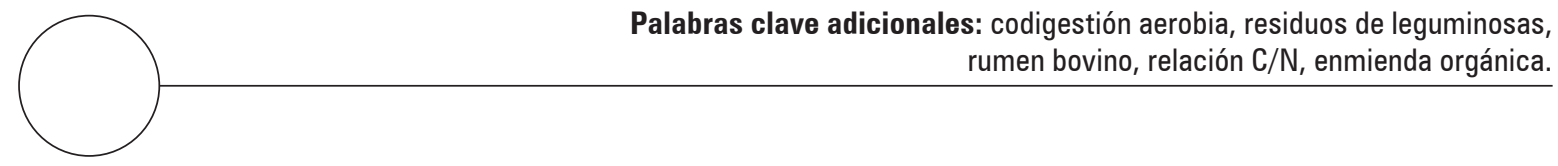

Facultad de Ingeniería, Ingeniería Ambiental, Grupo de Investigación INTEGRA, Tecnológico de Antioquia - Institución Universitaria (TdeA), Medellín (Colombia).

2 Autor para correspondencia: sarangoo1@tdea.edu.co 


\section{ABSTRACT}

This paper presents the physicochemical and microbiological analysis of the compost of legume waste and bovine rumen, both individually and combined, obtained by aerobic digestion. The independent variable was the percentage by mass of legume residues, producing four treatments: 0, 25, 50 and 100\%. The source of legume waste was obtained from bean pods 58\%, vetch $39 \%$ and kidney-bean $3 \%$ in wet mass. The rumen was achieved from bovine stomach lavage shortly after slaughter in a slaughterhouse. Temperature, $\mathrm{pH}$, moisture, ash, density, conductivity, water holding capacity (WHC), cation exchange capacity (CEC) and elements such as organic C, N, K, Ca, Mg y $\mathrm{Zn}$ were analyzed. The lowest carbon/nitrogen $(\mathrm{C} / \mathrm{N})$ relation of co-composting was in the waste legume-bovine rumen mixture (50-50\%) with $28.51 \%$, while the single plant legume compost was 9.14 . The parameters of mass percentage of $\mathrm{N}, \mathrm{Mg}$ and $\mathrm{Zn}$; as well as the CEC and conductivity showed the highest values in the vegetable compost, without the presence of Salmonella, making it a potential organic amendment providing such nutrients to the soil in any crop that requires it.

Additional key words: aerobic codigestion, legume waste, bovine rumen, $\mathrm{C} / \mathrm{N}$ ratio, organic amendments.

Fecha de recepción: 09-06-2016 Aprobado para publicación: 24-11-2016
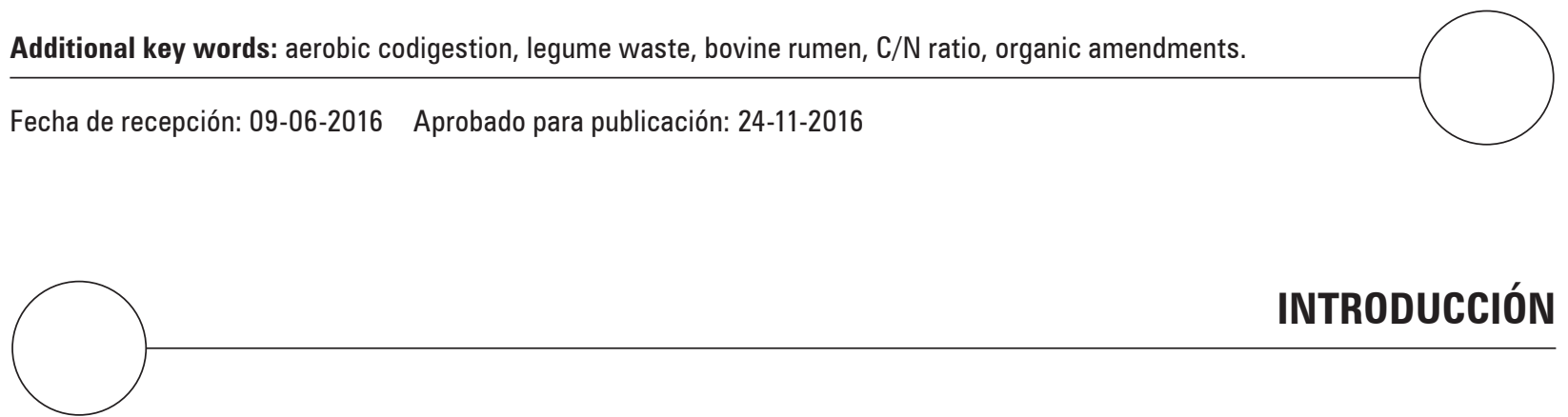

Diversos problemas ambientales de origen antrópico se presentan actualmente en el planeta que afectan el suelo, el agua y la atmósfera, tales problemáticas no solo impactan el hábitat natural (McLellan et al., 2014), sino que tienen consecuencias para los seres humanos en el corto y largo plazo, afectando muchos países, en especial los más pobres (CELAM, 2007). El más grave en un amplio umbral de tiempo es el cambio climático, originado por una cadena de fenómenos en efecto dominó (IPCC, 2013). Siendo el primero de ellos la emisión de gases llamados de efecto invernadero (GEI), los cuales ocasionan el efecto invernadero y como consecuencia el incremento de la temperatura en la tropósfera, fenómeno llamado calentamiento global (Arango et al., 2012), con efectos severos sobre los cultivos (Fischer et al., 2016).

Entre los principales GEI se encuentran el dióxido de carbono $\left(\mathrm{CO}_{2}\right)$, el metano $\left(\mathrm{CH}_{4}\right)$ y los óxidos de nitrógeno en especial el $\mathrm{N}_{2} \mathrm{O}$ (IPCC, 2013). La principal fuente en el mundo de $\mathrm{CH}_{4}$ es la ganadería, especialmente la proveniente de los bovinos (WBCSD y WRI, 2004). La segunda fuente en importancia de emisión de $\mathrm{CH}_{4}$ son los rellenos sanitarios que generan, por digestión anaerobia, $\mathrm{CO}_{2}, \mathrm{CH}_{4}$ y lixiviados, estos últimos como resultado de procesos simultáneos de escorrentía, infiltración y lixiviación de los productos de descomposición bioquímica (Pellón-Arrechea et al., 2015).
El incremento desproporcionado de generación de residuos y la dificultad de encontrar y aplicar soluciones ambientales para su mitigación, hacen del compostaje una opción muy usada para el tratamiento de residuos sólidos orgánicos (RSO), aprovechándolos para producir un acondicionador orgánico de suelos por digestión aerobia (Sepúlveda y Alvarado, 2013). El compostaje por codigestión aerobia es un proceso fisicoquímico y microbiológico que permite combinar diferentes sustratos o RSO en biorreactores aerobios, por aireación forzada o natural obteniendo un acondicionador de suelos (Negro et al., 2001; Rynk et al., 1992), dicho proceso sirve tanto para la productividad de la agricultura y la ganadería como para la mitigación del $\mathrm{CH}_{4}$ como GEI, evitando que los $\mathrm{RSO}$ vayan a un relleno sanitario. Sin embargo el término codigestión ha sido más utilizado por la literatura para el proceso de degradación anaerobio, que para el aerobio (Arango et al., 2012).

Se han realizado múltiples estudios de co-compostaje por codigestión aerobia combinando gran diversidad de sustratos orgánicos, subproductos de la caña: cachaza, bagazo y vinaza (Bohórquez et al., 2014); pulpa de café, bovinaza, gallinaza, banano de rechazo y hojarasca (Escobar et al., 2013); residuos orgánicos urbanos (Cabeza et al., 2013); hojas de almendro con estiércol bovino (Suleiman et al., 2015) y combinación de residuos de frutas entre otros (Nasreen y Iqbal, 2012). 
En general la materia orgánica está compuesta principalmente por carbono $(\mathrm{C})$ y nitrógeno $(\mathrm{N})$, y en el nivel celular está presente el fósforo (P). El C abunda en las partes leñosas de las plantas, como tronco, paja, aserrín, ramas y en la ruminaza. Es el principal formador de la lignina, celulosa y los azúcares (Marqués y Urquiaga, 2005). El N por su parte predomina en las partes verdes de las plantas, algunos restos de comida, césped, excrementos de animales incluyendo la ruminaza (Muñoz et al., 2010; Marqués y Urquiaga, 2005). Actualmente se ha incrementado la demanda mundial de nitrógeno para fertilización superando 100.000.000 t. El P por su parte se extrae de roca fosfórica, la cual está muy limitada; por lo que es pertinente que tanto para el $\mathrm{N}$ como para el $\mathrm{P}$ se hagan intentos por proteger no solo sus vertimientos en recursos hídricos, sino también extraer de ellos, al máximo, de lixiviados o biomasa agrícola y ganadera (Muñoz et al., 2010). Esto ha sido una de las razones principales que han impulsado la realización del presente estudio. Se ha elegido la leguminosa como biomasa clave por su capacidad para fijar nitrógeno en su raíz, gracias a la presencia de bacterias que pueden producir la enzima nitrogenasa, la cual rompe el enlace covalente triple del nitrógeno molecular, transformando este gas presente en el aire e incorporándolo al suelo en diferentes iones como el nitrato y el amonio (Whitten et al., 2011; Calvo-García, 2011).

Además de los anteriores elementos también se deben evaluar otros como el potasio (K), calcio (Ca), magnesio $(\mathrm{Mg})$ y zinc $(\mathrm{Zn})$ entre otros, como lo estipula normatividad colombiana (NTC 5167). Un compost maduro puede aplicarse como una enmienda orgánica de carácter sólida (Icontec, 2004).

El objetivo de la presente investigación fue la caracterización de compostajes a partir de codigestión aerobia de residuos de ruminaza y leguminosa.

\section{METODOLOGÍA}

\section{Procedimiento experimental}

El proceso de co-compostaje se realizó por combinación de biomasa vegetal y animal. La fuente vegetal estuvo representada por vainas de leguminosa tomadas de una mezcla aleatoria de fríjol al 58\%, arveja al $39 \%$ y habichuela al 3\% en masa húmeda, pesadas 12 $\mathrm{h}$ después de haberse extraído los granos. La fuente animal fue ruminaza, obtenida del lavado estomacal
2 h después del sacrificio del ganado en una planta industrial frigorífica.

La unidad de análisis usada como variable independiente fue el porcentaje en masa de vainas de leguminosa en cada tratamiento para realizar los respectivos compostajes. El diseño experimental completamente aleatorio (Tab. 1) fue estimado para el porcentaje de leguminosa con base en la serie $25\left(2^{\mathrm{n}}\right)$, incluyendo el 0, obteniéndose 25, 50 y 100\%, llevado a cabo en el vivero del Tecnológico de Antioquia - Institución Universitaria, Medellín.

Tabla 1. Diseño experimental de codigestión aerobia.

\begin{tabular}{|l|c|c|c|}
\hline \multicolumn{1}{|c|}{$\begin{array}{c}\text { Tratamientos } \\
\text { de compostaje }\end{array}$} & $\begin{array}{c}\text { Variable } \\
\text { \% vegetal } \\
\text { (leguminosa) }\end{array}$ & Repeticiones & $\begin{array}{c}\text { Total } \\
\text { ensayos }\end{array}$ \\
\hline Animal I (A) & 0 & 5 & 5 \\
\hline Animal y vegetal I (AVI) & 25 & 5 & 5 \\
\hline $\begin{array}{l}\text { Animal y vegetal II } \\
\text { (AVII) }\end{array}$ & 50 & 5 & 5 \\
\hline Vegetal I (V) & 100 & 5 & 5 \\
\hline
\end{tabular}

Se pesaron $5,5 \mathrm{~kg}$ de muestra para cada tratamiento vegetal y animal, $6 \mathrm{~kg}$ para las mezclas de co-compostaje entre ambos, y se llevaron a compostadores plásticos de $20 \mathrm{~L}$ con tapa, completando de esta forma 20 unidades experimentales de compostaje.

Cada semana se realizó dos veces volteo para garantizar la oxigenación y una vez por semana se adicionaron $300 \mathrm{~mL}$ de agua desionizada para hidratar la biomasa (Rodríguez et al., 2013); las celdas permanecían tapadas para protegerlas de la luz solar. Después de dos meses de proceso aerobio se tomaron muestras de compost de cada unidad experimental para su respectivo análisis en el laboratorio.

\section{Métodos analíticos}

La temperatura $(\mathrm{T})$ y el $\mathrm{pH}$ se midieron con un equipo digital ST3100-F con sonda ST310 y la conductividad eléctrica (CE) con sonda STCON3 (Ohaus, Parsippany, NJ). La $\mathrm{T}$ y $\mathrm{pH}$ se monitorearon durante $60 \mathrm{~d}$ en cada una de las 20 unidades de compostaje. Al final del proceso se realizó la caracterización fisicoquímica y microbiológica de los diferentes compostajes. El N total fue medido por el método Kjeldahl, el carbono orgánico (CO) mediante oxidación química por el método NTC 5167 (Icontec, 2004) y el P mediante 
fotometría; los metales potasio (K), calcio (Ca), magnesio $(\mathrm{Mg})$ y zinc $(\mathrm{Zn})$ se cuantificaron mediante electroforesis capilar; la capacidad de retención de agua (CRA), humedad $(\mathrm{H})$ y cenizas $(\mathrm{Cz})$ por gravimetría mediante método NTC 5167; finalmente la capacidad de intercambio catiónico (CIC) por volumetría según método NTC 5167. A los datos obtenidos se les realizó un análisis de comparación entre pares de medias mediante distribución t-Student, asumiendo un nivel de significancia si $P \leq 0,05$ (Ceballos-Urrego, 2014).

\section{RESULTADOS Y DISCUSIÓN}

\section{Análisis fisicoquímico}

Los resultados de caracterización realizados en el laboratorio se muestran en las figuras 1 a 8 y en general están dados en porcentaje en masa. Un primer factor muy importante en el proceso de compostaje es la humedad $(\mathrm{H})$, ya que permite la movilidad de los microorganismos, el transporte de nutrientes y el flujo de oxígeno para mantener las condiciones aerobias (Kulikowska y Gusiatin, 2015). En la figura 1 se muestra un contenido de $\mathrm{H}$ alto, por encima del $80 \%$ en todos los tratamientos, resultado esperado debido a la hidratación semanal suministrada y a la producción de moléculas de $\mathrm{H}_{2} \mathrm{O}$ en la reacción bioquímica de codigestión aerobia; este resultado es comparable con la humedad de compost obtenido a partir de estiércol de cerdo y estiércol de residuos lácteos con un 81 y 84\%, respectivamente (Qian et al., 2014). El contenido de $\mathrm{Cz}$ presentó diferencias significativas en todos los tratamientos, el valor más alto se encuentra en el compostaje vegetal con un $25,52 \pm 0,01 \%$ y más bajo en el compostaje animal-vegetal al $50 \%$ de cada uno (AVII), con un $14,39 \pm 0,01 \%$. El CO no presentó diferencias significativas en dos de los cuatro tratamientos, de acuerdo a la figura 1 , para A y AVI, con valores de $35,78 \pm 1,26$ y $35,92 \pm 1,15 \%$, mientras que Vy AVII si poseen diferencias significativas $(P \leq 0,05)$ entre síy con respecto a los demás, con valores de $33,18 \pm 0,81$ y $40,46 \pm 1,60 \%$ respectivamente, siendo las concentraciones mínima y máxima en los tratamientos; esta última es similar para compostajes a partir de estiércol de cerdo y vaca con valores de 42,2 $\pm 0,45$ y $41,1 \pm 1,22 \%$ respectivamente, y ligeramente superior al compost de paja de arroz con $38,73 \pm 0,99 \%$ (Oian et al., 2014). Con respecto a la norma (NTC 5167) cumple para el CO, ya que supera el $30 \%$, mientras que el contenido de $\mathrm{H}$ supera el $20 \%$, como máximo establecido, debería entonces reducírsele el contenido de agua para así cumplir este parámetro normativo (Icontec, 2004).

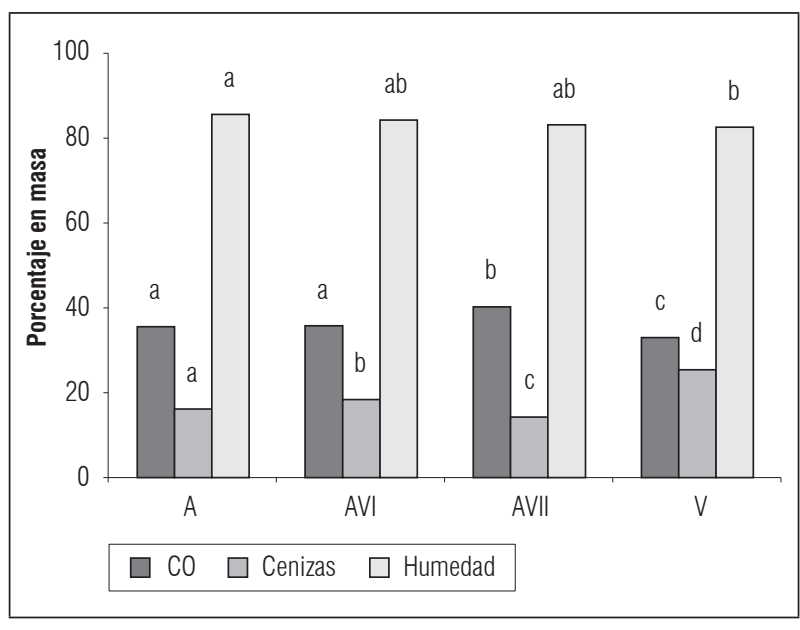

Figura 1. Contenido de humedad, cenizas y carbono orgánico (CO) en compostajes. Promedios con letras distintas, para el mismo parámetro, indican diferencia significativa según la prueba t-Student.

Las concentraciones de $\mathrm{N}, \mathrm{P}, \mathrm{K}$ y Ca, expresadas como $\mathrm{N}$ total, $\mathrm{P}_{2} \mathrm{O}_{5}, \mathrm{~K}_{2} \mathrm{O}$ y $\mathrm{CaO}$, respectivamente, poseen diferencias significativas $(P \leq 0,05)$, excepto para A y AVII del P (figura 2). Se observa que el $\mathrm{N}$ contenido en el compost vegetal es mayor con un valor de $3,63 \pm 0,57 \%$, lo cual se explica debido a su contenido de $100 \%$ leguminosa y su capacidad de fijación de este elemento en la raíz, y menor en AVII con un $0,52 \pm 0,07 \%$. El $\mathrm{P}_{2} \mathrm{O}_{5}$ fue mayor en el tratamiento AVII con $0,760 \pm 0,08 \%$ y menor en el vegetal con $0,588 \pm 0,007 \%$, esto deriva en una relación másica de $\mathrm{N} / \mathrm{P}$ de 14,12 para solo leguminosa y 2,73 para solo ruminaza, siendo este último superior a un valor de 1,0 en estudios realizados en España para estiércol de ganado vacuno (Muñoz et al., 2010). El valor para leguminosa al 100\% (V) es poco más del doble con respecto al valor inicial de heces de ganado tomadas de pastizales de la pampa argentina sin compostar con una relación N/P de 6,25 (Semmartin, 2006). Estos átomos de $\mathrm{N}$ y $\mathrm{P}$ presentes en las moléculas orgánicas de muchas rutas metabólicas aerobias y anaerobias poseen una concentración siempre inferior al C, como se puede evidenciar en estos análisis.

El catión potasio $\left(\mathrm{K}^{+}\right)$es un macronutriente esencial para el crecimiento y desarrollo de los cultivos. Algunos de los procesos bioquímicos de las plantas, donde 


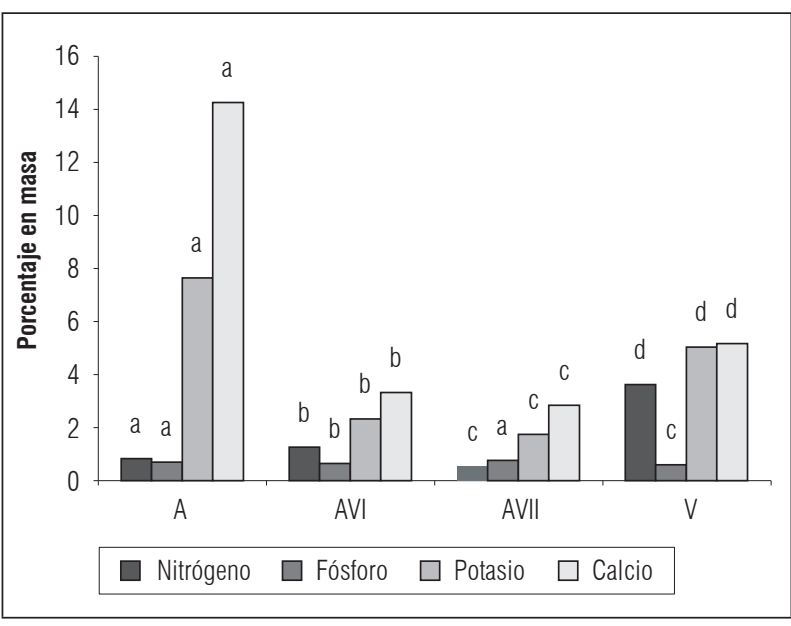

Figura 2. Concentraciones de N, P, K y Ca en compostajes. Promedios con letras distintas, para el mismo elemento, indican diferencia significativa según la prueba t-Student.

el $\mathrm{K}^{+}$está presente son: osmorregulación, síntesis de almidones, activación enzimática, síntesis de proteínas, movimiento estomático y balance de cargas iónicas (Maathuis y Sanders, 1994). Los resultados obtenidos para este metal alcalino, expresado como $\mathrm{K}_{2} \mathrm{O}$, muestran que está presente en una mayor proporción en el compost animal con 7,66 $\pm 0,73 \%$, mientras que es mucho menor en el AVII con $1,74 \pm 0,16 \%$.

El catión calcio $\left(\mathrm{Ca}^{2+}\right)$, por su parte, interacciona por fuerzas de atracción electrostáticas, con los grupos fosfato con carga negativa de las cabezas polares de los fosfolípidos, reduciendo las fuerzas de repulsión entre estos grupos, aumentando de esta forma el empaquetamiento de los fosfolípidos, dando como resultado una disminución de la fluidez de la membrana celular (Feduchi et al., 2014). El contenido de calcio expresado como $\mathrm{CaO}$ supera en todos los tratamientos la concentración de otros elementos, debido a su mayor disponibilidad en los suelos de Antioquia, hallándose en mayor cantidad en el compost animal con un valor de $14,30 \pm 0,54 \%$ y menor en el AVII con un valor de 2,84 $\pm 0,21 \%$. Tanto la concentración de $\mathrm{K}_{2} \mathrm{O}$ como la de $\mathrm{CaO}$ superan las obtenidas para cachaza fresca, con 0,735 y $6,52 \%$, respectivamente, este es un subproducto obtenido en la industria azucarera y en trapiches paneleros, con potencial como enmienda orgánica para suelos en cultivos de fríjol y maíz (Forero et al., 2008).

La relación másica carbono/nitrógeno $(\mathrm{C} / \mathrm{N})$ posee diferencias significativas $(P \leq 0,05)$ (Fig. 3$)$. Esta relación es muy importante para determinar la calidad de

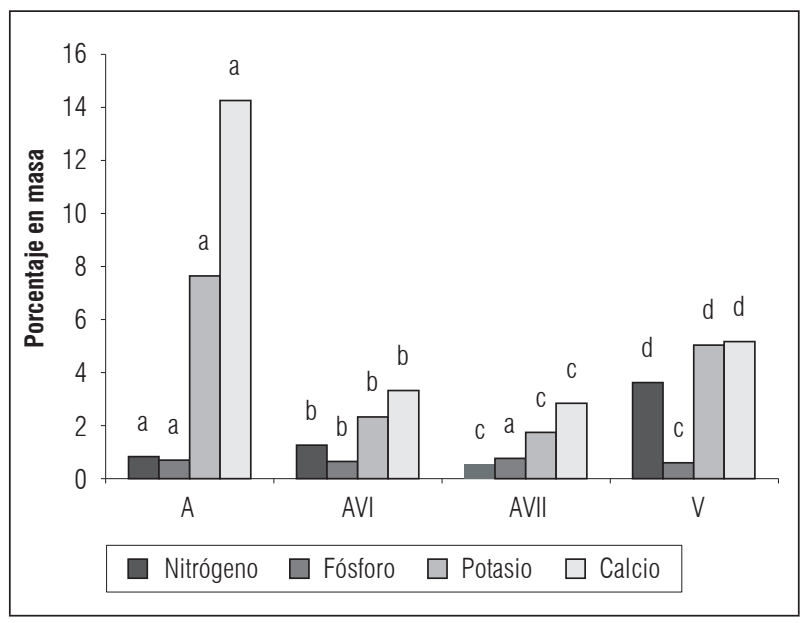

Figura 3. Relación másica carbono/nitrógeno en compostajes. Promedios con letras distintas indican diferencia significativa según la prueba t-Student.

un compost, ya que influye en la biomasa bacteriana (Negro et al., 2001). El valor más alto se encuentra en el compost AVII $(77,81)$, seguido de A $(43,11)$, AVI $(28,51)$ y el menor valor se tiene para el compost $\mathrm{V}$ $(9,14)$, siendo estos dos últimos más cercanos a una relación de 15 como valor óptimo de referencia (Sepulveda y Alvarado, 2013). Otros autores han obtenido valores de 128,4 para compostajes de solo leguminosa y 71 y 60 para mezclas de este con residuos orgánicos municipales (Cabeza et al., 2013). Los valores de compostajes V y AVI pueden usarse como acondicionadores orgánicos y fuente de $\mathrm{N}$ al suelo en el cultivo que lo requiera (Kulikowska y Gusiatin, 2015).

En rutas metabólicas de bioquímica vegetal existen enzimas que contienen zinc ( $\mathrm{Zn}$ ), como la ARN polimerasa y la anhidrasa carbónica (AC). Existe una relación directa entre la concentración de $\mathrm{Zn}$ y la actividad de la AC, si disminuye el $\mathrm{Zn}$ baja su actividad y en consecuencia disminuye el fenómeno físico-bioquímico de la fotosíntesis. La enzima AC está presente en el estroma de los cloroplastos y facilita la trasferencia molecular de $\mathrm{CO}_{2} / \mathrm{HCO}_{3}$ para la captura del $\mathrm{CO}_{2}$ atmosférico, realizando así una importante labor para la mitigación del calentamiento global desde las hojas de las plantas (Lizarazo et al., 2013).

El Zn y el Mg forman parte de los metales de interés en los procesos de intercambio iónico y conductividad eléctrica, poseen diferencias significativas $(P \leq 0,05)$ (Fig. 4). El Zn está presente en una mayor concentración en el compost vegetal $(\mathrm{V})$, con un valor de 
$0,383 \pm 0,004 \%$; la menor concentración se obtuvo en el animal con un valor de $0,121 \pm 0,010 \%$, este valor es incluso casi tres veces superior al obtenido en compost a partir de lodos de plantas de tratamiento de aguas residuales (Kulikowska y Gusiatin, 2015) y seis veces superior al obtenido en compost a partir de bagazo de la industria azucarera (Mohee et al., 2015). El Mg que forma parte de la clorofila (Whitten et al., 2011), se encontró en menor concentración en el AVII con un valor de $0,528 \pm 0,015 \%$ y su mayor concentración en el compost vegetal, con un valor de $1,173 \pm 0,113 \%$, favoreciendo, en comparación con otros, la actividad fotosintética y por tanto la captura de carbono, clave para las estrategias de mitigación del efecto invernadero y el calentamiento global (IPCC, 2013).

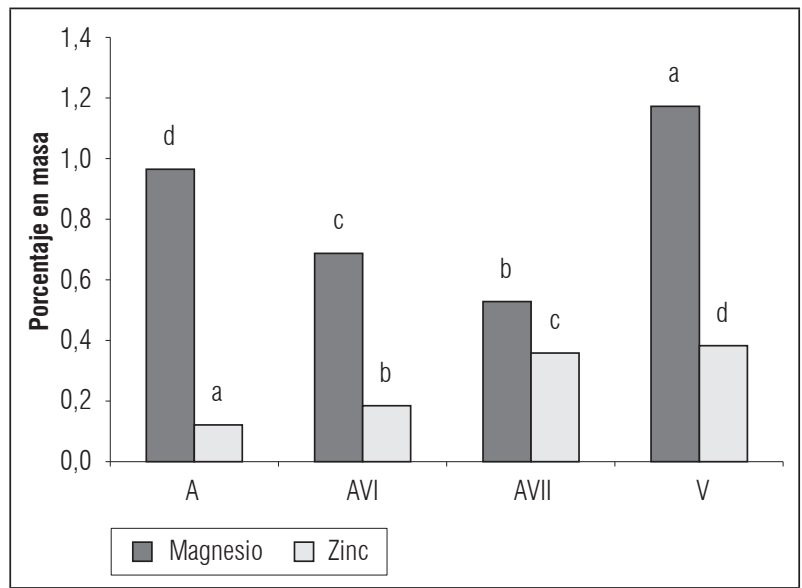

Figura 4. Contenido de $\mathrm{Mg}$ y $\mathrm{Zn}$ en compostajes. Promedios con letras distintas, para el mismo elemento, indican diferencia significativa según la prueba t-Student.

La capacidad de intercambio catiónico (CIC) está dada en miliequivalentes por cada $100 \mathrm{~g}$ de compost y presenta diferencias significativas según la figura 5 . El valor mayor se obtuvo en el compost vegetal con un valor de 44,48 $\pm 0,00 \mathrm{meq} / 100 \mathrm{~g}$, y el valor inferior se obtuvo con el tratamiento animal con $27,55 \pm 1,95$ meq/100 g. Referente a la relación de la capacidad de intercambio catiónico sobre el carbono orgánico (CIC/CO), el valor superior fue obtenido en el compost vegetal con $134 \mathrm{meq} / 100 \mathrm{~g}$ CO y es el único que presenta diferencias significativas $(P \leq 0,05)$.

La capacidad de retención de agua (CRA) que representa la cantidad de agua que puede contener el compost con relación a su masa seca, se indica en la figura 6. Todos los valores son relativamente altos, solo los tratamientos A y AVII no presentaron diferencias

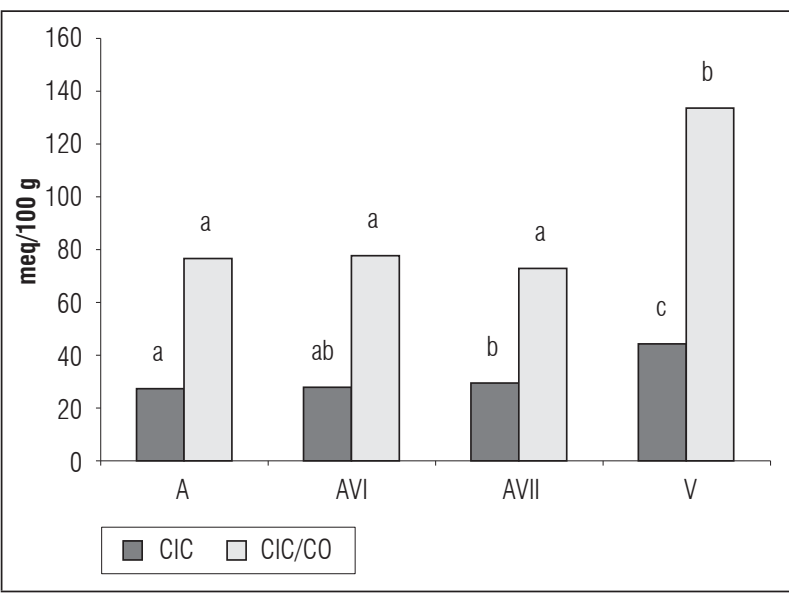

Figura 5. Relación de capacidad de intercambio catiónico $\mathrm{CIC}$ en meq/100 g y CIC/CO en meq/100 g CO en compostajes. Promedios con letras distintas indican diferencia significativa según la prueba t-Student.

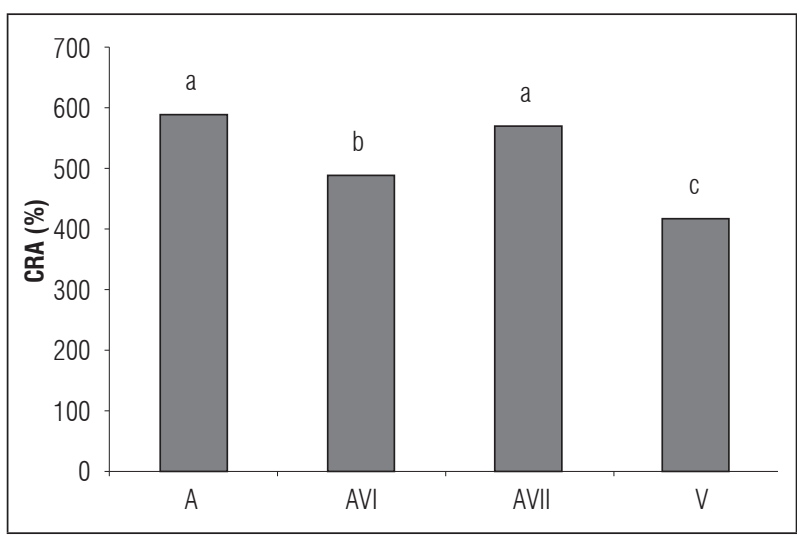

Figura 6. Capacidad de retención de agua (CRA). Promedios con letras distintas indican diferencia significativa según la prueba t-Student.

significativas; el mayor se obtuvo en el compost animal con un $589,33 \pm 26,61 \%$, lo que indica que el compost puede absorber agua en casi seis veces su masa seca, el menor valor se obtuvo para el compost vegetal con un $417,69 \pm 9,19 \%$.

La densidad de los compostajes realizados (Fig. 7) dio como resultado valores más altos para A, AVI y AVII $\left(0,1 ; 0,11\right.$ y $\left.0,11 \mathrm{~g} \mathrm{~cm}^{-3}\right)$, solo los tratamientos AVI y AVII no presentaron diferencias significativas. El valor más bajo se encuentra en el compost vegetal $(0,06$ $\mathrm{g} \mathrm{cm}^{-3}$ ), debido a la contextura del mismo, pastosa, porosa y poco compacta. 


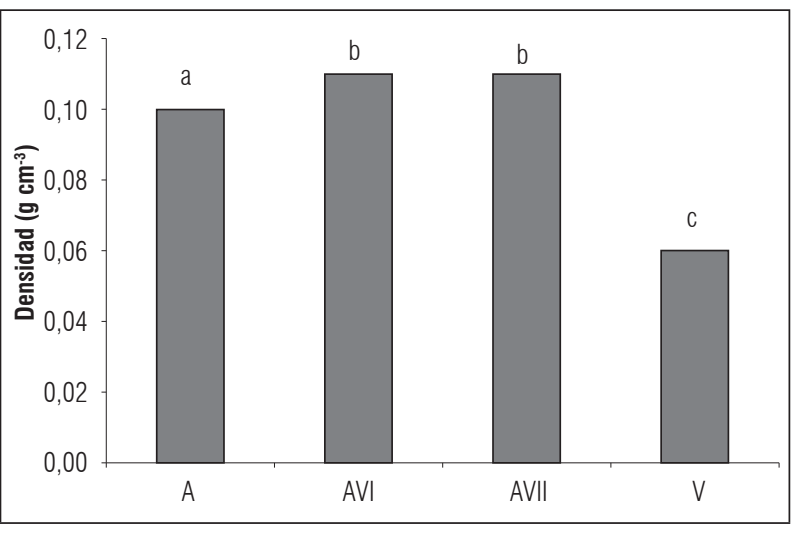

Figura 7. Valores de densidad de los compostajes. Promedios con letras distintas indican diferencia significativa según la prueba t-Student.

La conductividad eléctrica (Fig. 8) presenta diferencias significativas $(P \leq 0,05)$, el valor sobresaliente está en el compost vegetal, 5,22 $\pm 0,04 \mathrm{dS} \mathrm{m}^{-1}$, debido principalmente a su alta capacidad de intercambio catiónico (CIC) (Fig. 5). El valor más bajo se tiene para el compost animal (A) con un valor de 1,51 $\pm 0,01 \mathrm{dS}$ $\mathrm{m}^{-1}$. Este valor es incluso superior al obtenido en compost a partir de bagazo de la industria azucarera, con $1,25 \pm 0,03 \mathrm{dS} \mathrm{m}^{-1}$ (Mohee et al., 2015). Solo el valor del compost vegetal supera lo recomendado por algunos autores: $<2 \mathrm{dS} \mathrm{m}^{-1} \mathrm{y}<4 \mathrm{dS} \mathrm{m}{ }^{-1}$ (Oviedo-Ocaña et al., 2014).

\section{Monitoreo de temperatura y $\mathrm{pH}$}

Los resultados de temperatura en todos los compostajes oscilaron entre 20 y $30^{\circ} \mathrm{C}$, sobrepasando

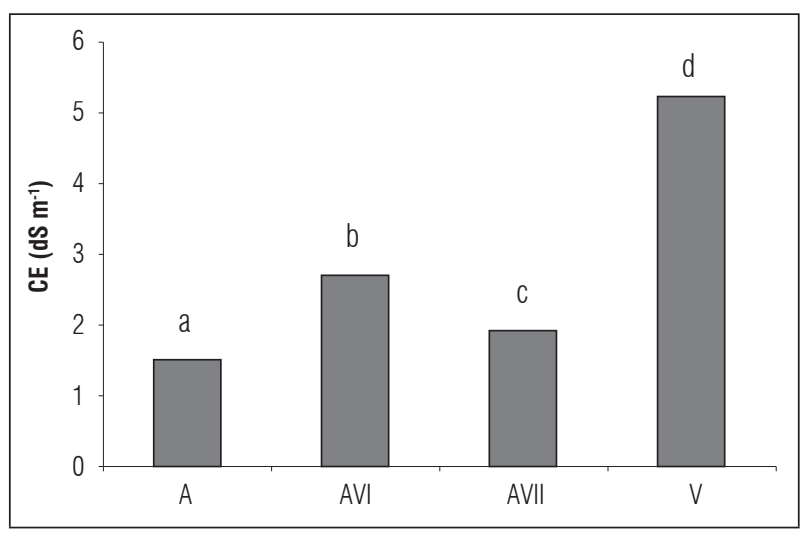

Figura 8. Valores de conductividad eléctrica en los compostajes. Promedios con letras distintas indican diferencia significativa según la prueba t-Student.

ligeramente la temperatura ambiente. Esto indica que durante los dos meses de la descomposición se operó en el rango de temperatura mesofílica y no termofílica como es de esperarse. Esto se puede explicar por el tamaño de los recipientes o celdas en los que se realizó la codigestión aerobia, $20 \mathrm{~L}$, los cuales se hallaban en un lugar abierto; este es un volumen pequeño para producir compost, por lo que la energía calórica generada en el metabolismo exotérmico de las bacterias aerobias se transfería hacia afuera de cada celda de compostación, conservando poco el calor, contrario a lo que ocurre en un compostaje a gran escala, dando como resultado una disminución de la temperatura.

El seguimiento al $\mathrm{pH}$ en función del tiempo se muestra en la figura 9. Durante los primeros $45 \mathrm{~d}$ es básico entre 7,1 y 9, pero después de este hasta el día

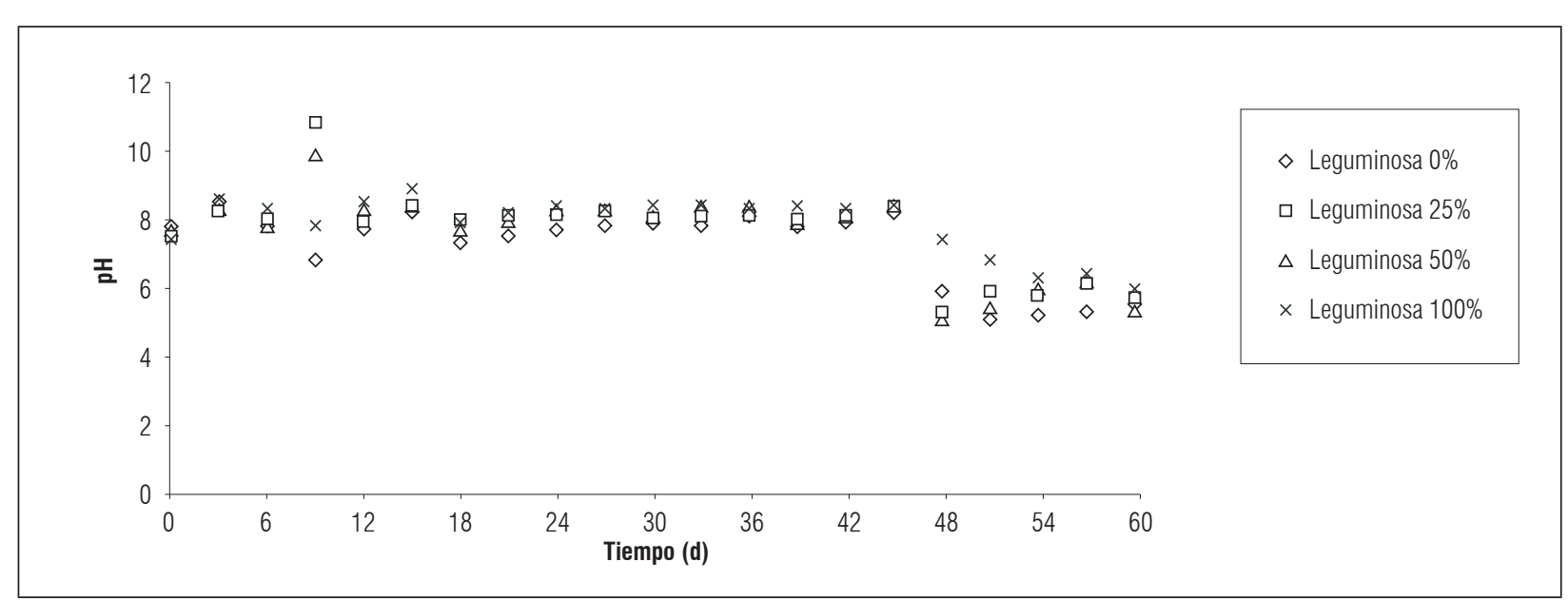

Figura 9. Series de pH en el tiempo para compostajes de leguminosa y rumen. 
60 disminuye llegando a valores de acidez entre 5 y 6,7. Para el caso de los tratamientos con leguminosa, la presencia de mayor contenido de $\mathrm{N}$ en la biomasa inicial pudo ocasionar el incremento del $\mathrm{pH}$ desde el inicio del proceso, debido a su metabolismo al transformarse en amoniaco y posterior ionización en amonio e hidroxilo (Negro et al., 2001). La caída del pH luego del día 45 puede obedecer a la liberación de todo el $\mathrm{NH}_{4}^{+}$, que se ha fugado como amoniaco, no teniendo ya el compost una fuente que pueda neutralizar la acidez ocasionada por los ácidos carboxílicos de cada corta, también llamados ácidos grasos volátiles (Aemiro et al., 2016). En compostajes realizados a partir de gallinaza y material celulósico se han obtenido de forma similar durante un periodo de $45 \mathrm{~d}$ una tendencia ascendente desde un $\mathrm{pH}$ de 7,8 hasta 10,2 (Hoyos et al., 2010). Sin embargo, los resultados del presente estudio convergen más hacia los obtenidos en los primeros $20 \mathrm{~d}$ en compostaje de bagazo de agave y biosólidos provenientes de una planta de tratamiento de vinazas tequileras (Rodríguez et al., 2013).

\section{Análisis microbiológicos}

Los tratamientos fueron analizados microbiológicamente, evaluando siete parámetros principales (Tab. 2). Según la normatividad colombiana, la ausencia de Salmonella determina su potencial uso como enmienda orgánica o acondicionador de suelo, en los análisis el resultado fue "ausente" en $25 \mathrm{~g}$ de compost final para todos los tratamientos, cumpliendo de esta forma con la NTC 5167 (Icontec, 2004). Las enterobacterias no se detectaron para los tratamientos de codigestión al 25 y $50 \%$, mientras que en los sustratos individuales sí hubo presencia, para el compost de solo rumen (AI) se obtuvo $10 \mathrm{UFC} \mathrm{g}^{-1}$, quedando dentro de la norma, sin embargo el tratamiento con $50 \%$ leguminosa (AVII) presentó un exceso de enterobacterias, 23.000 UFC g ${ }^{-1}$, muy superior al valor máximo (1.000 UFC g-1) establecido por la NTC 5167. Dada la temperatura, siempre inferior a $30^{\circ} \mathrm{C}$, se obtuvo en todos los tratamientos mayor presencia de microorganismos mesófilos que termófilos al final del proceso. Los mohos estuvieron muy por debajo de estos, mientras que las levaduras solo se detectaron en el compost vegetal al 50\% (AVII). Los nemátodos o protozoos estuvieron ausentes en todos los tratamientos de compost al final del proceso.

\section{CONCLUSIONES}

La relación carbono/nitrógeno $(\mathrm{C} / \mathrm{N})$ más cercana a valores óptimos se encuentran en los compostajes vegetal-animal al $25-75 \%$ (AVI) y en el vegetal al 100\% $(\mathrm{V})$, con 28,51 y 9,14 , respectivamente. La presencia de $\mathrm{P}$ en los compostajes es baja, el valor mayor está en AVII con $0,76 \%$. El porcentaje de Ca supera en la mayoría de los tratamientos la concentración de otros elementos, hallándose en mayor cantidad en el compost animal con un valor de $14,30 \%$. Mientras que el potasio está presente en una mayor proporción en el compost animal con $7,66 \%$.

Tales resultados fisicoquímicos, sumados a la sanidad microbiológica de los compost al 0, 25 y $50 \%$ de leguminosa, hacen que estos según la norma NTC 5167 puedan ser aplicados como enmiendas orgánicas.

\section{REFERENCIAS BIBLIOGRÁFICAS}

Aemiro, A., M. Hanada, K. Umetsu y T. Nishida. 2016. The effect of Sunphenon 30S-O on methane emission, nutrient intake, digestibility and rumen fermentation. Anim. Feed Sci. Technol. 214, 34-43. Doi: 10.1016/j. anifeedsci.2016.02.007

Arango, S., J. Montoya y G. Peñuela. 2012. Effect assessment of temperature on biogas production from bovine rumen and banana (Musa acuminata) stem undergoing anaerobic codigestion. Int. J. Environ. Eng. 4(3/4), 197-209. Doi: 10.1504/IJEE.2012.050794

Bohórquez, A., Y. Puentes y J. Menjivar. 2014. Evaluación de la calidad del compost producido a partir de subproductos agroindustriales de caña de azúcar. Cienc.

Tabla 2. Evaluación microbiológica de los compost obtenidos en los tratamientos de codigestión aerobia.

\begin{tabular}{|l|c|c|c|c|c|c|}
\hline \multirow{2}{*}{ Tratamiento } & Mesófilos & Termófilos & Mohos & Levaduras & Entero bacterias & Salmonella en 25g \\
\cline { 2 - 7 } & UFC g-1 $^{-1}$ & UFC g & UFC g & UFC g-1 & UFC g & (CS) \\
\hline Al & $8,0 \times 10^{-10}$ & $6,3 \times 10^{8}$ & $3,0 \times 10^{3}$ & 0,0 & $1,0 \times 10^{1}$ & Ausente \\
\hline $\mathrm{VI}$ & $3,9 \times 10^{9}$ & $7,1 \times 10^{8}$ & $2,4 \times 10^{4}$ & 0,0 & 0,0 & Ausente \\
\hline $\mathrm{AVI}$ & $5,8 \times 10^{9}$ & $3,0 \times 10^{7}$ & $4,0 \times 10^{3}$ & $1,0 \times 10^{1}$ & 0,0 & Ausente \\
\hline $\mathrm{AVII}$ & $1,3 \times 10^{9}$ & $3,4 \times 10^{8}$ & $4,0 \times 10^{1}$ & 0,0 & $2,3 \times 10^{4}$ & Ausente \\
\hline
\end{tabular}


Tecnol. Agropecu. 15(1), 73-81. Doi: 10.21930/rcta. vol15_num1_art:398

Cabeza, I., R. López, M. Ruiz-Montoya y M. Díaz. 2013. Maximising municipal solid waste e legume trimming residue mixture degradation in composting by control parameters optimization. J. Environ. Manag. 128, 266273. Doi: 10.1016/j.jenvman.2013.05.030

Calvo-García, S. 2011. Bacterias simbióticas fijadoras de nitrógeno. Cuad. Tomás 3, 173-186.

Ceballos-Urrego, L. 2014. Lecciones de estadística. Tecnológico de Antioquia, Medellín, Colombia.

CELAM. 2007. V Conferencia General del Episcopado Latinoamericano y del Caribe. Discípulos y Misioneros de Jesucristo para que nuestros pueblos en Él tengan vida "Yo soy el Camino, la Verdad y la Vida (Jn 14,6). Documento conclusivo. CELAM, Brasil: En: http://www. celam.org/aparecida/Espanol.pdf, consulta: enero de 2016.

Escobar, N., J. Mora y N. Romero. 2013. Respuesta agronómica de Zea mays L. y Phaseolus vulgaris L. a la fertilización con compost. Luna Azul 37, 18-29.

Feduchi, E., C. Romero, E. Yáñez, I. Blasco y C. García-Hoz. 2014. Bioquímica. $2^{\text {a }}$ ed. Panamericana, Madrid.

Fischer, G., F. Ramírez y F. Casierra-Posada. 2016. Ecophysiological aspects of fruit crops in the era of climate change. A review. Agron. Colomb. 34(2), 190-199. Doi: 10.15446/agron.colomb.v34n2.56799

Forero, F., J. Torres, y H. Balaguera. 2008. Efecto de la aplicación de cachaza fresca y de dos sistemas de producción de maíz y maíz con asocio fríjol sobre las propiedades físicas de un Inceptisol. Rev. Colomb. Cienc. Hortíc. 2(2), 205-216. Doi: 10.17584/rcch.2008v2i2.1188

Hoyos, J., C. Vargas y R. Velasco. 2010. Evaluación de compost obtenido en pila móvil empleando mezclas de gallinaza de jaula con material celulósico. Rev. Fac. Cienc. Agropecu. 8(1), 54-60.

Icontec. 2004. NTC 5167. Norma técnica colombiana. Productos para la industria agrícola. Productos orgánicos usados como abonos o fertilizantes y enmiendas de suelo. Instituto Colombiano de Normas Técnicas y Certificación, Bogotá.

IPCC. 2013. Climate change 2013. The physical science basis. Contribution of Working Group I to the Fifth Assessment Report of the Intergovernmental Panel on Climate Change. Intergovernmental Panel on Climate Change. Cambridge University Press, Cambridge, UK.

Kulikowska, D. y Z. Gusiatin. 2015. Sewage sludge composting in a two-stage system: Carbon and nitrogen transformations and potential ecological risk assessment. Waste Manag. 38, 312-320. Doi: 10.1016/j. wasman.2014.12.019
Lizarazo, M., C. Hernández, G. Fischer y M. Gómez. 2013. Biomasa, parámetros foliares y sintomatología en respuesta a diferentes niveles de manganeso, zinc y boro en curuba (Passiflora tripartita var. mollissima). Rev. Colomb. Cienc. Hortíc. 7(1), 31-45. Doi: 10.17584/ rcch.2013v7i1.2033

Maathuis, F. y D. Sanders. 1994. Mechanism of high affinity potassium uptake in roots of Arabidosis thaliana. Proc. Natl. Acad. Sci. 91, 9272-9276. Doi: 10.1073/ pnas.91.20.9272

Marqués, M. y R. Urquiaga. 2005. Manual del buen compostador. Grupo de Acción para el Medio Ambiente, Madrid.

McLellan, R., L. Iyengar, B. Jeffries y N. Oerlemans (eds.). 2014. Informe planeta vivo 2014: Personas y lugares, especies y espacios. World Wide Fund for Nature (WWF), Gland, Suiza.

Mohee, R., A. Boojhawon, B. Sewhoo, S. Rungasamy, G. Somaroo y A. Mudhoo. 2015. Assessing the potential of coal ash and bagasse ash as inorganic amendments during composting of municipal solid wastes. J. Environ. Manag. 159, 209-217. Doi: 10.1016/j.jenvman.2015.05.008

Muñoz, N., B. Gómez, C. Rico, J. Rico, J. Fernández y J. Renedo. 2010. Procesos físico-biológico-químico para eliminar materia orgánica: nitrógeno y fósforo de estiércol de vacuno lechero. pp. 225-234. En: Memorias Ecofarm, II Congreso Español de Gestión Integral de Deyecciones Ganaderas. Barcelona, España.

Nasreen, Z. y J. Iqbal. 2012. Lab scale composting of fruits and vegetable waste at elevated temperature and forced aeration. Pak. J. Zool. 44(5), 1285-1290,

Negro, M., F. Villa, J. Aibar, R. Aracón, P. Ciria, M. Cristábal, C. Zaragoza, A. Benito, A. García, G. García, C. Labrador, D. Lacasta, J. Lezaún, R. Meco, G. Pardo, M. Solano, C. Tornery C. Zaragoza. 2001. Producción y gestión del compost. Informaciones Técnicas 88. En: http:// digital.csic.es/bitstream/10261/16792/1/2000\%20 Compost\%20CIEMAT.pdf; consulta: enero de 2016.

Oviedo-Ocaña, E., P. Torres-Lozada y L. Marmolejo-Rebellon. 2014. evaluation of the addition of wood ash to control the ph of substrates in municipal biowaste composting. Ing. Investig. Tecnol. 15(3), 469-478.

Pellón-Arrechea, A., M. López-Torres, M. Espinosa-Lloréns y O. González-Díaz. 2015. Propuesta para tratamiento de lixiviados en un vertedero de residuos sólidos urbanos. Ing. Hidrául. Ambient. 36(2), 3-16.

Qian, X., G. Shen, Z. Wang, C. Guo, Y. Liu, Z. Lei y Z. Zhang. 2014. Co-composting of livestock manure with rice straw: Characterization and establishment of maturity evaluation system. Waste Manag. 530535. Doi: 10.1016/j.wasman.2013.10.007

Rodríguez, R., J. Jiménez, J. Real, E. Salcedo, J. Zamora y G. Íñiguez. 2013. Utilización de subproductos de la 
industria tequilera. Parte 11. Compostaje de bagazo de agave crudo y biosólido provenientes de una planta de tratamiento de vinazas tequileras. Rev. Int. Contam. Ambient. 29(4), 303-313.

Rynk, R., M. Van de Kamp, G. Wilson, M. Single, T. Richard, J. Colega, F. Gouin, L. Laliberty, K. Day, D. Murphy, A. Hoitink y W. Brinton. 1992. On-farm composting handbook. Northeast Regional Agricultural Engineering Service, Ithaca, NY.

Semmartin, M. 2006. Dinámica de la descomposición y la mineralización neta del nitrógeno y del fósforo de heces de vacunos en pastoreo sobre un pastizal templado. Rev. Arg. Prod. Anim. 26, 193-202.

Sepúlveda, L. y J. Alvarado. 2013. Manual de aprovechamiento de residuos orgánicos a través de sistemas de compostaje y lombricultura en el Valle de Aburrá. Oficina Asesora de Comunicaciones del Área Metropolitana del Valle de Aburrá. Medellín, Colombia.

Suleiman, A., E. Aiyelari e I. Otene. 2015. Effects of tillage and Terminalia catappa L. leaf compost on soil properties and performance of Capsicum chinense Jacq. Int. J. Adv. Agric. Res. 3, 73-82.

WBCSD y WRI. 2004. The greenhouse gas protocol. Genova: World Business Council Sustainable Development y World Resources Institute. En: http://pdf.wri.org/ ghg_protocol_2004.pdf; consulta: diciembre de 2013.

Whitten, K., R. Davis, M. Peck y G. Stanley. 2011. Química. $8^{a}$ ed. Cengage Learning, México. 